\title{
CHEMMEDCHEM
}

CHEMISTRY ENABLING DRUG DISCOVERY

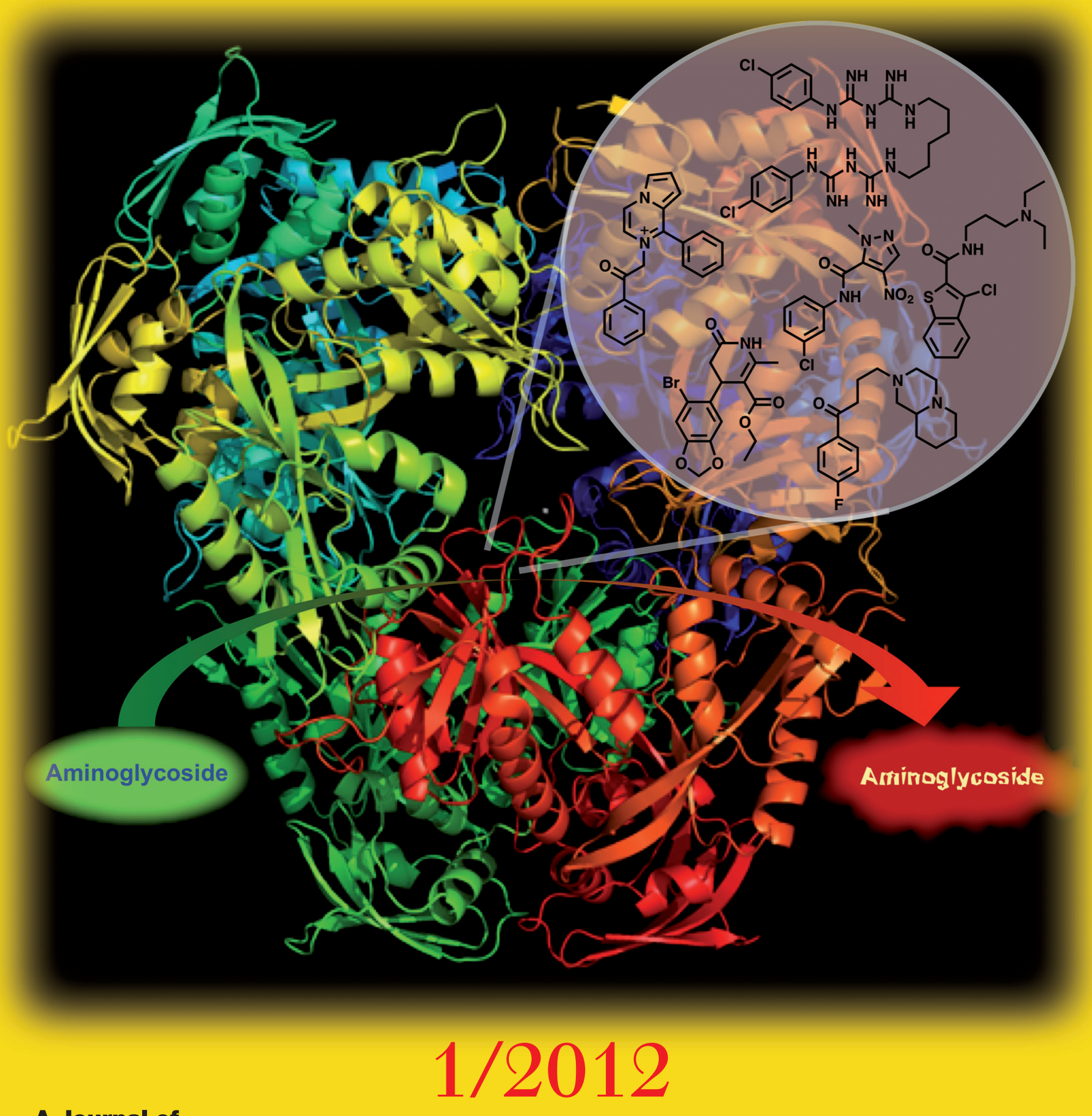

\section{A Journal of}

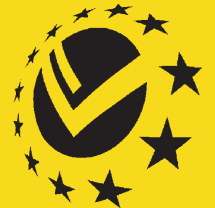

ChemPubSoc Europe

The back cover picture shows Eis, the enzyme that converts aminoglycoside antibiotics from their active (green) to their inactive (red) form, a process that causes drug resistance in tuberculosis (TB). The compounds shown are the first examples of Eis inhibitors, which could find application in combination therapies with aminoglycosides to treat TB. For more details, please see the Communication by Sylvie GarneauTsodikova et al. on p. $73 \mathrm{ff}$. 


\section{Back Cover Picture}

\section{Keith D. Green, Wenjing Chen, and Sylvie Garneau-Tsodikova*}

The back cover picture shows Eis, the enzyme that converts aminoglycoside antibiotics from their active (green) to their inactive (red) form, a process that causes drug resistance in tuberculosis (TB). The compounds shown are the first examples of Eis inhibitors, which could find application in combination therapies with aminoglycosides to treat TB. For more details, please see the Communication by Sylvie Garneau-Tsodikova et al. on p. $73 \mathrm{ff}$.

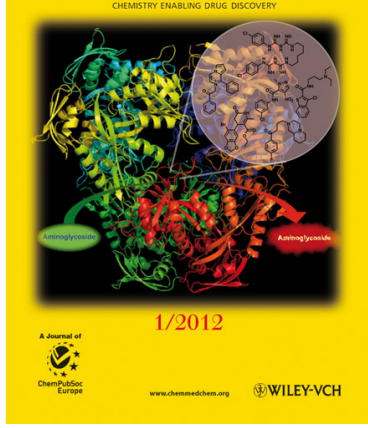

